\title{
Melatonin enhances salt tolerance by promoting MYB108A-mediated ethylene biosynthesis in grapevines
}

\author{
Lili Xu', Guangqing Xiang ${ }^{1}$, Qinghua Sun², Yong Ni², Zhongxin Jin', Shiwei Gao ${ }^{1}$ and Yuxin Yao ${ }^{1}$
}

\begin{abstract}
The signal molecules melatonin and ethylene play key roles in abiotic stress tolerance. The interplay between melatonin and ethylene in regulating salt tolerance and the underlying molecular mechanism of this interplay remain unclear. Here, we found that both melatonin and 1-aminocyclopropane-1-carboxylic acid (ACC, a precursor of ethylene) enhanced the tolerance of grapevine to $\mathrm{NaCl}$; additionally, ethylene participated in melatonin-induced salt tolerance. Further experiments indicated that exogenous treatment and endogenous induction of melatonin increased the ACC content and ethylene production in grapevine and tobacco plants, respectively. The expression of MYB108A and ACS1, which function as a transcription factor and a key gene involved in ethylene production, respectively, was strongly induced by melatonin treatment. Additionally, MYB108A directly bound to the promoter of ACS1 and activated its transcription. MYB108A expression promoted ACC synthesis and ethylene production by activating ACS1 expression in response to melatonin treatment. The suppression of MYB108A expression partially limited the effect of melatonin on the induction of ethylene production and reduced melatonin-induced salt tolerance. Collectively, melatonin promotes ethylene biosynthesis and salt tolerance through the regulation of ACS1 by MYB108A.
\end{abstract}

\section{Introduction}

Soil salinization is an important environmental problem, and salt has become one of the most commonly encountered abiotic stresses that affects fruit crops, including grapevines, causing nutritional imbalance, ion toxicity, osmotic stress and oxidative damage and severely reducing crop growth, yield and fruit quality. Globally, $\sim 830$ million ha of land is affected by salinization, and this area is anticipated to increase in the near future ${ }^{1}$. Grapevines are widely cultivated worldwide and are ranked as sensitive or moderately sensitive to salt stress ${ }^{2}$. It is difficult to obtain highly salt-tolerant grapevine cultivars through traditional breeding methods; in contrast,

\footnotetext{
Correspondence: Yuxin Yao (yaoyx@sdau.edu.cn)

'State Key Laboratory of Crop Biology, Collaborative Innovation Center of Fruit \& Vegetable Quality and Efficient Production, College of Horticulture Science and Engineering, Shandong Agricultural University, Tai-An, Shandong 271018, China

${ }^{2}$ State Key Laboratory of Crop Biology, College of Life Science, Shandong Agricultural University, Tai-An, Shandong 271018, China
}

genetic modification is an effective approach for the creation of new crop varieties with improved characteristics. However, more experimental data are needed to reveal salt tolerance mechanisms that allow crops to optimize their responses to salt. Grapevines and other plants have evolved multiple strategies for protection against salt, one of which is hormone-guided tolerance to salt, including melatonin and ethylene ${ }^{3,4}$. Additionally, plant responses to abiotic stresses are controlled by the interactive hormonal network ${ }^{5}$; plant hormone crosstalk can occur at the level of the regulation of hormone biosynthesis, signal transduction or common target gene expression $^{6}$.

Ethylene is an important signaling molecule mediating numerous important biological processes, including responses to abiotic stresses ${ }^{7}$. The action of ethylene depends on its concentration in cells and the sensitivity of plants to this hormone ${ }^{8}$. Ethylene biosynthesis is primarily regulated by 1 -aminocyclopropane-1-carboxylate (ACC)

\section{(c) The Author(s) 2019}

(c) (i) Open Access This article is licensed under a Creative Commons Attribution 4.0 International License, which permits use, sharing, adaptation, distribution and reproduction cc) in any medium or format, as long as you give appropriate credit to the original author(s) and the source, provide a link to the Creative Commons license, and indicate if changes were made. The images or other third party material in this article are included in the article's Creative Commons license, unless indicated otherwise in a credit line to the material. If material is not included in the article's Creative Commons license and your intended use is not permitted by statutory regulation or exceeds the permitted use, you will need to obtain permission directly from the copyright holder. To view a copy of this license, visit http://creativecommons.org/licenses/by/4.0/. 
synthase (ACS) and ACC oxidase (ACO) at the transcriptional or posttranslation levels ${ }^{9}$. Salt promotes ethylene production in various species by modulating the activity of ACS and $\mathrm{ACO}^{10,11}$. Additionally, the endogenous overproduction of ethylene or exogenous treatment with the ethylene precursor ACC enhances the tolerance of some plants, including Arabidopsis ${ }^{12}$ and maize $^{13}$, to salt stress. The inhibition of ACS expression reduces salt tolerance in the wild tomato species Solanum chilense $e^{14}$. In addition to ethylene synthesis, transduction through ethylene responsive factors (ERFs) may also have crucial functions in the plant responses to salt stresses ${ }^{15}$; in addition to the response to salt, ethylene also plays a key role in other stresses, including cold stress in grapevines ${ }^{16}$ and drought stress in soybean ${ }^{17}$. Although substantial progress has been made in identifying the roles of ethylene in abiotic stress responses, more studies are needed to unravel the precise control of ethylene production and signaling in response to abiotic stresses.

In a recent study, we demonstrated that melatonin promoted ethylene biosynthesis and that MYB108A and $A C S 1$ were strongly induced by melatonin treatment during grape berry ripening ${ }^{18}$. Melatonin (N-acetyl-5methoxytryptamine) is an indoleamine that is synthesized from L-tryptophan metabolism via serotonin. Melatonin is a pleiotropic and highly conserved molecule and is ubiquitous in animals and plants ${ }^{19}$. Melatonin, as a plant regulator, is stress inducible, and both exogenous application and endogenous induction play a key role in enhancing plant tolerance to salt and other abiotic stresses ${ }^{4,20}$. The beneficial role of melatonin in the stress response is broadly attributable to the regulation of the gene expression involved in abiotic stress responses $^{19-21}$. Additionally, there is significant crosstalk between melatonin and other plant growth regulators, including abscisic acid (ABA), jasmonic acid, and salicylic acid ${ }^{22}$; it has been shown that exogenous melatonin application enhances drought priminginduced cold tolerance and drought tolerance by modulating ABA levels in barley and apple, respectively ${ }^{23,24}$. Melatonin also interacts with other signaling pathways; recent studies have reported that mitogen-activated protein kinase pathways (MAPKs) are required for melatonin-mediated defense responses in plants ${ }^{25}$; nitric oxide is required for melatonin-enhanced tolerance against salt stress in rapeseed seedlings ${ }^{26}$.

To date, the molecular pathways associated with crosstalk between melatonin and other signals remain largely unknown. The objectives of this study were to elucidate whether melatonin can regulate salt tolerance via ethylene signals and to reveal the underlying molecular mechanism by which melatonin enhances ethylene biosynthesis via MYB108A and ACS1 in grapevines.

\section{Results}

Melatonin enhances salt tolerance of 'Crimson seedless' grapevines partially through ethylene

To investigate whether melatonin and ethylene enhanced tolerance to salt, grapevines were watered with $100 \mathrm{mM} \mathrm{NaCl}$ to induce salt stress. After three weeks of $\mathrm{NaCl}$ treatment, the grapevines were markedly withered, and the leaves showed necrotic phenotypes; in contrast, the grapevines watered with $\mathrm{NaCl}$ plus melatonin or ACC (precursor of ethylene) showed slight marginal necrosis and exhibited less severe phenotypes (Fig. 1a). Additionally, analyses of the vine height increment, relative electrical conductivity and root activity also indicated that the addition of melatonin or ACC alleviated injury by $\mathrm{NaCl}$ (Fig. 1b-d). Therefore, ACC and melatonin treatment enhanced the tolerance of vines to $\mathrm{NaCl}$.

Grapevine leaves completely withered when treated with $\mathrm{NaCl}$ plus melatonin and aminovinylglycine (AVG, inhibitor of ethylene synthesis) at 24 DAT; in contrast, vine leaves still appeared green, although they exhibited symptoms of marginal necrosis under treatment of $\mathrm{NaCl}$ plus melatonin (Fig. 1a). Additionally, the grapevines treated with $\mathrm{NaCl}$ plus melatonin and AVG exhibited a lower vine height increment and root activity and a higher electrical conductivity of leaves than those treated with $\mathrm{NaCl}$ plus melatonin (Fig. 1b-d). Therefore, AVG decreased the function of melatonin in enhancing vine tolerance to $\mathrm{NaCl}$, indicating that melatonin enhanced vine tolerance to $\mathrm{NaCl}$, partially through ethylene.

\section{Melatonin increases ethylene production in the roots and leaves of 'Crimson seedless' grapevines and tobacco plants}

To further investigate the influence of melatonin on ethylene, exogenous melatonin treatment was performed by watering 'Crimson seedless' vine roots. The $50 \mu \mathrm{M}$ melatonin treatment significantly enhanced the melatonin levels in vine roots and leaves (Fig. 2a). As a result, the content of ACC and the ethylene production rate were significantly enhanced in the melatonin-treated roots and leaves compared to the control tissues (Fig. 2b, c). Additionally, grape acetylserotonin methyltransferase ( $V v i A S M T)$, the final enzyme in the melatonin biosynthesis pathway ${ }^{27}$, was ectopically expressed to increase the biosynthesis of endogenous melatonin in tobacco plants. The transformation was confirmed by PCR detection of the target gene (Fig. 2d), and lines 1 and 3, with different levels of $V v i A S M T$ expression, were selected for further assays (Fig. 2e). VviASMT ectopic expression enhanced the melatonin content of roots and leaves in the tobacco transgenic lines (Fig. 2f). The two transgenic plants exhibited significantly higher ACC and ethylene production rates than the control (Fig. $2 \mathrm{~g}$, h). Therefore, ethylene production is enhanced by melatonin by both exogenous treatments and the induction its synthesis in planta. 

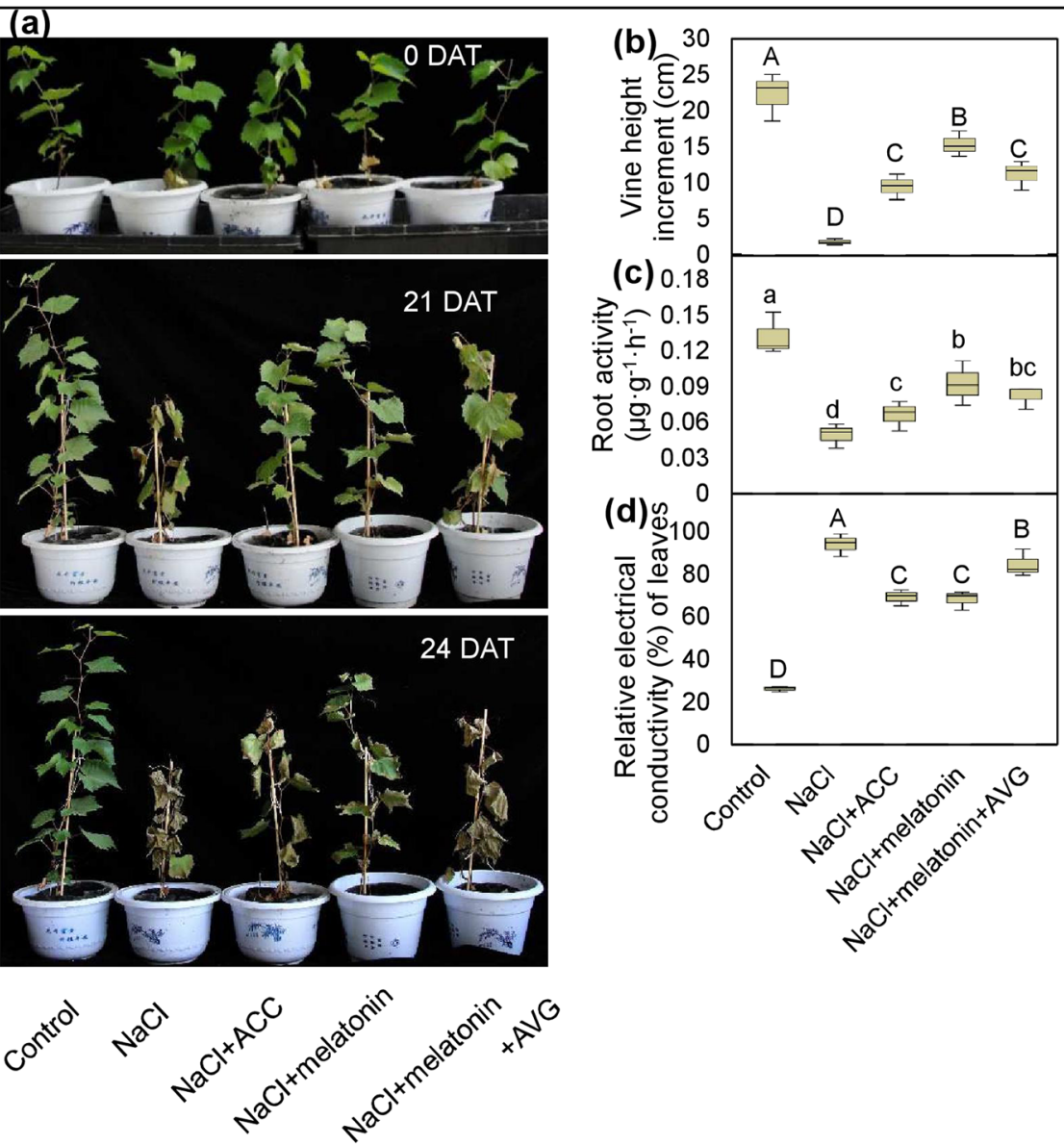

Fig. 1 Effects of the addition of ACC, melatonin and melatonin with AVG on the tolerance of 'Crimson seedless' grapevines to salt. a Phenotypes of grapevines grown in a greenhouse under natural conditions and under various treatments at 0, 21, and 24 DAT. b-d Vine height increment $\mathbf{b}$, root activity $\mathbf{c}$ and leaf relative electrical conductivity $\mathbf{d}$ under various treatments at 21 DAT. Vine cuttings were treated with $100 \mathrm{mM}$ $\mathrm{NaCl}$ in the presence or absence of $50 \mu \mathrm{M}$ melatonin, $50 \mu \mathrm{M}$ ACC and $2 \mu \mathrm{M}$ AVG. DAT, days after treatment; ACC, 1-aminocyclopropane-1-carboxylic acid; AVG, aminovinylglycine. Values represent the means \pm SD of three replicates. Values indicated by the same lowercase and capital letters are not significant at $P<0.05$ and $P<0.01$, respectively, on the basis of Duncan's multiple range test

Melatonin induces the expression of MYB108A and ACS1, which function as a transcription factor and an ethylene biosynthesis gene, respectively, in 'Crimson seedless' grapevine roots and/or calluses

Our previous work demonstrated that MYB108A (VIT_205s0077g00500) and ACS1 (VIT_215s0046g 02220 ), identified in a previous study ${ }^{28}$ and by sequence alignment with its counterpart in Arabidopsis (Fig. S1), respectively, were significantly upregulated by melatonin treatment in berries based on RNA-Seq analysis ${ }^{18}$. To further dissect their expression response to melatonin, qRT-PCRs were performed in roots at different time points after melatonin treatment. MYB108A and ACS1 were gradually induced by melatonin and reached a maximum at $72 \mathrm{~h}$, with expression levels that were 13.75and 3.07-fold higher, respectively, in the treated roots than in the control (Fig. 3a, b). Additionally, histochemical
GUS staining and GUS activity were analyzed in the transformed grape calluses expressing the $M Y B 108 A$ promoter::GUS $\left(P_{M Y B 108 A}:: G U S\right)$ construct. For transformants with $P_{M Y B 108 A}:: G U S$, calluses treated with melatonin produced clearly stronger GUS staining and significantly higher GUS activity than calluses without melatonin treatment (Fig. 3c, d). Therefore, MYB108A and $A C S 1$ were transcriptionally induced by melatonin in vine roots.

To detect whether MYB108A possessed transcription factor activity, its subcellular location and transcriptional activation properties were determined. The fusion protein MYB108A::GFP was observed in the nucleus of the epidermal cells of onion and tobacco leaves (Fig. 4a, d), while the GFP signal was detected in membrane, cytoplasm and nucleus in the control cells (Fig. 4b, c). Therefore, MYB108A was located in the nucleus. On the 

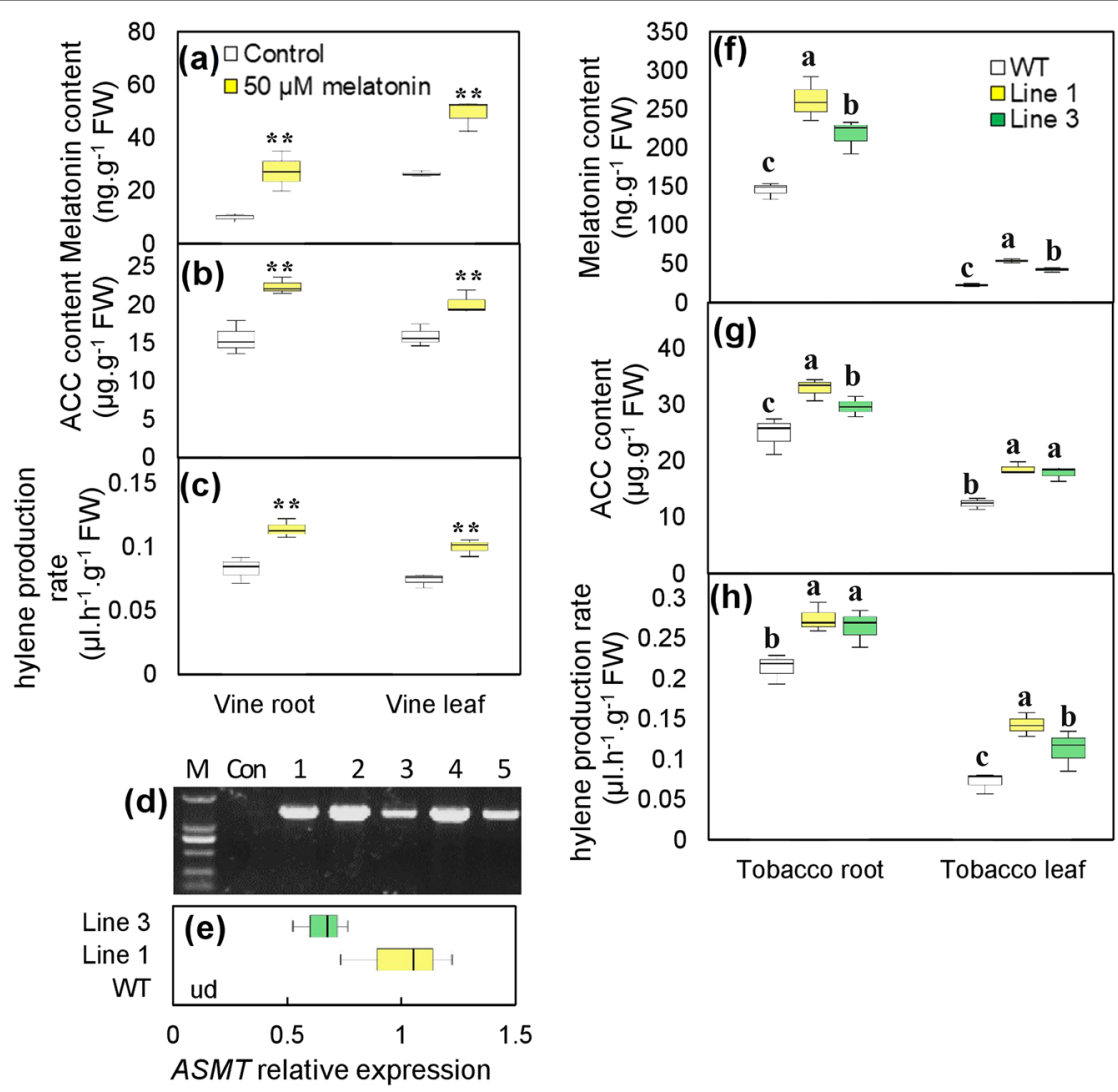

Fig. 2 Effects of exogenous application and endogenous induction of melatonin on the ACC content and ethylene production. $\mathbf{a}-\mathbf{c}$ Changes in the content of melatonin $\mathbf{a}$ and ACC $\mathbf{b}$ and ethylene production rate $\mathbf{c}$ in 'Crimson seedless' vine roots and leaves at 3 days after $50 \mu \mathrm{M}$ melatonin treatment. $\mathbf{d}$ PCR identification of ASMT-overexpressing tobacco plants using DNA templates and specific primers from 355 and ASMT. e ASMT expression in tobacco leaves of wild-type (WT) and transgenic lines. $\mathbf{f}-\mathbf{h}$ Changes in melatonin $\mathbf{f}$, ACC content $\mathbf{g}$ and ethylene production rate $\mathbf{h}$ in leaves and roots of WT and transgenic tobacco plants. The numbers represent 5 different transgenic lines of tobacco plants in d. ud, undetectable; WT, wild type; M, DNA marker; Con, control; ASMT, acetylserotonin methyltransferase. Values represent the means \pm SD of three replicates. ${ }^{* *}$ highly significant difference, $P<0.01$. Values indicated by the same lowercase letters are not significant at $P<0.05$. A one-way analysis of variance (ANOVA) followed by a nonparametric Kruskal-Wallis test was employed

other hand, full-length $M Y B 108 A$ was subcloned into the pGBKT7 vector containing a GAL4 DNA-binding domain, and this construct was transformed into yeast. Positive X-gal activity was observed in yeast containing pGBKT7-MYB108A but not in yeast containing pGBKT7 on the screening medium (Fig. 4e). These results indicated that MYB108A could activate the expression of the reporter gene in the yeast system. Additionally, the $102 \mathrm{C}$-terminal amino acids were responsible for transcription activation (Fig. 4e). Taken together, these results indicate that MYB108A is a nuclear transcription factor.

To determine whether ACS1 is a key enzyme controlling ACC biosynthesis, suppressor 1 and suppressor 2 were produced by transforming antisense cDNA fragments matching the ACS1 3-UTR into grape calluses. Compared with the control, the two suppressors had reduced ACS1 transcript levels, ACC contents and ethylene production rates (Fig. $4 \mathrm{f}-\mathrm{h}$ ); therefore, $A C S 1$ functions in ACC synthesis.
MYB108A binds to the promoter of ACS1 and activates its transcription in yeast, grapevine calluses and tobacco leaves

A yeast one-hybrid assay was performed to determine whether the MYB108A protein was able to bind MBS element (AACCTAA, Fig. S3) in the promoter of ACS1. The MBS element or mutant MBS element (mMBS) was inserted into the pAbAi vector, and the corresponding constructs were designated pAbAi-MBS and pAbAimMBS, respectively. The complete coding region of $M Y B 108 A$ was cloned into the yeast expression vector pGADT7. The resulting pGADT7-MYB108A and pGADT7 constructs were transformed into the yeast strain Y1HGold carrying either pAbAi-MBS or pAbAimMBS. All transformed yeast cells were grown on medium without leucine (Leu) and uracil (Ura) to confirm the successful transformation (Fig. 5a). As expected, only the yeast clones containing pAbAi-MBS and pGADT7-MYB108A grew on synthetic dropout medium 

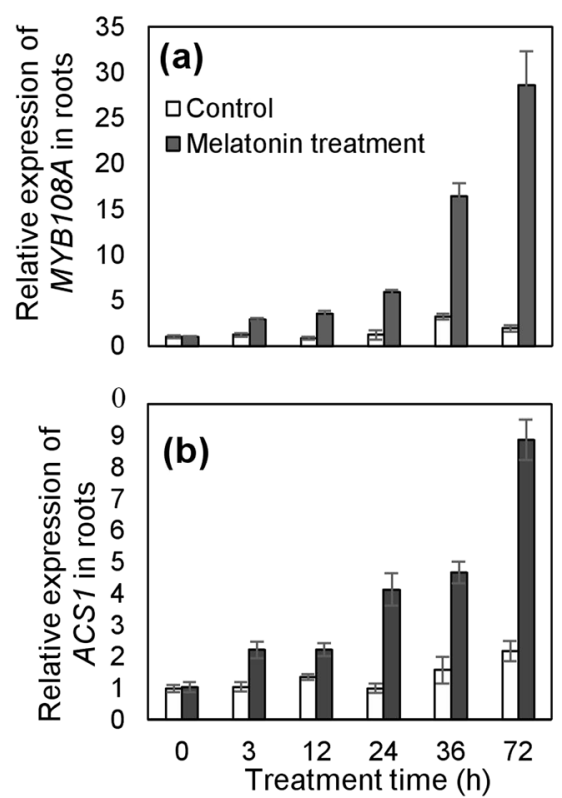

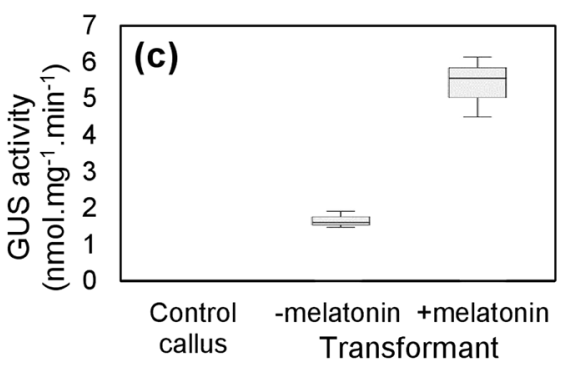

(d)

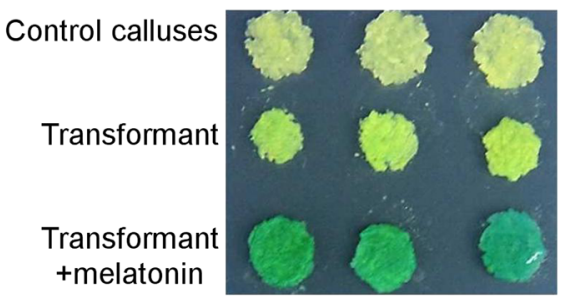

Fig. 3 Expression responses of MYB108A and ACS1 determined by qRT-PCR. $\mathbf{a}, \mathbf{b}$ and/or GUS activity $\mathbf{c}$ and staining $\mathbf{d}$. qRT-PCRs were performed in the roots of 'Crimson seedless' in vitro shoot cultures at different time points $\mathbf{a}, \mathbf{b}$. GUS activity $\mathbf{c}$ and staining $\mathbf{d}$ were analyzed using 'Crimson seedless' calluses expressing the MYB108A promoter:.GUS (transformant) construct. In this experiment, 50 mM melatonin was employed. In a-c, values represent the means \pm SD of 3 replicates. Photos of GUS staining were taken from the three replicates in $\mathbf{d}$

(SD/-Leu/-Ura) containing $500 \mu \mathrm{g} / \mathrm{L}$ AbA (Fig. 5a), indicating that MYB108A bound to the MBS binding site and activated transcription in the yeast system.

To further verify the direct binding of MYB108A to the MBS-containing recognition site in the ACS1 promoter, an electrophoretic mobility shift assay (EMSA) was performed with an oligo-probe containing an MBS element using purified recombinant His-MYB108A fusion protein. This indicated that specific DNA-MYB108A protein complexes were detected when the oligo-probe containing the MBS element was used. The formation of these complexes was gradually reduced with the application of increasing amounts of the unlabeled MBS competitor probe with the same sequence (Fig. 5c). In contrast, this competition was not detected when the mutated competitor was used (Fig. 5c). Therefore, the MYB108A protein was able to specifically bind to the MBS element of the ACS1 promoter.

To investigate whether MYB108A activated gene expression by interacting with the MBS element in plant cells, Agrobacterium-mediated transient expression of the GUS reporter gene in grape calluses was performed. The calluses cotransformed with MBS-35S mini-GUS and 35S-MYB108A were bluer in color and showed higher GUS activity than calluses transformed with mMBS-35S mini-GUS and 35S-MYB108A or only MBS-35S miniGUS (Fig. $5 \mathrm{~d}$, e). Therefore, the GUS gene was activated in grape calluses via the interaction between MYB108A and the MBS element. Additionally, the tobacco leaves cotransformed with the $\mathrm{P}_{\mathrm{ACS} 1}:$ ACS1-GUS (ACS1-GUS fusion gene driven by the ACS1 promoter) and 35S:: MYB108A, and those transformed with only $\mathrm{P}_{\mathrm{ACS} 1}:$ : ACS1-GUS were obtained. The results showed that transcripts of ACS1 and GUS activity were positively regulated by MYB108A (Fig. 5f, g). Therefore, MYB108A acts upstream of $A C S 1$ to activate its transcript levels.

\section{Melatonin promotes ethylene production by increasing MYB108A expression in 'Cabernet Sauvignon' grapevine leaves}

The fusion gene MYB108A-GUS driven by the $M Y B 108 A$ promoter was transiently expressed in grape leaves to demonstrate whether $M Y B 108 A$ can promote ethylene production in response to melatonin. The leaves expressing proMYB108A-GUS revealed increased GUS staining, GUS activity and $M Y B 108 A$ expression compared to the control (Fig. 6a-c). Additionally, the leaves expressing proMYB108A-GUS exhibited high ACS1 expression, a high ACC content and a high ethylene production rate (Fig. 6d-f). Therefore, MYB108A activated $A C S 1$ expression and thereby promoted ACC and ethylene biosynthesis in grapevine leaves.

Additionally, the melatonin-treated leaves expressing proMYB108A-GUS exhibited higher MYB108A expression, bluer color and higher GUS activity than the leaves without melatonin treatment (Fig. $6 \mathrm{a}-\mathrm{C}$ ), indicating that $M Y B 108 A$ was transcriptionally induced by melatonin. Additionally, the role of $M Y B 108 A$ in regulating $A C S 1$ expression and ACC and ethylene production was 


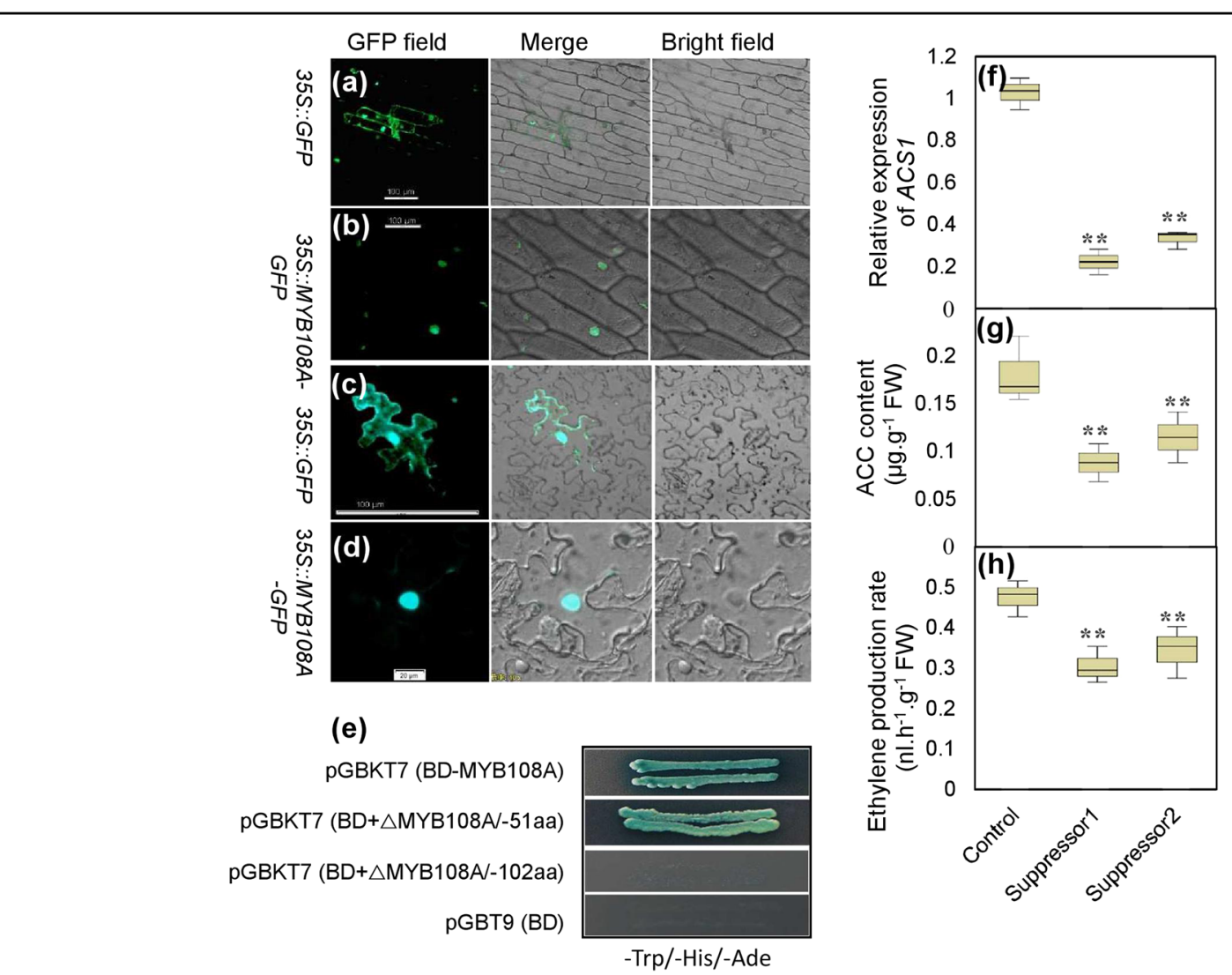

Fig. 4 Identification of the properties of MYB108A as a transcription factor. a-e and the function of ACS1 in the synthesis of ACC and ethylene f-h. 355::GFP and 355::MYB108A-GFP vectors were transiently expressed in onion $\mathbf{a}, \mathbf{b}$ and tobacco $\mathbf{c}$, $\mathbf{d}$ epidermis cells. GFP fluorescence, bright field, and overlay merge of bright field and fluorescent illumination are shown a-d. In e, BD indicates the binding domain; $\triangle$ MYB108A/-51aa and $\triangle \mathrm{MYB108A}$-102aa indicate the deletion of 51 and 102 C-terminal amino acids of the MYB108A protein, respectively. In $\mathbf{f}-\mathbf{h}$, suppressor 1 and suppressor 2 represent ACS1-suppressed 'Crimson seedless' calluses; values are presented as the means \pm SD of 3 replicates; ** highly significant difference on the basis of Duncan's multiple range test at $P<0.01$

strengthened by melatonin treatment (Fig. 6d-f). Moreover, the expression of ACS1 and ethylene production were significantly reduced in the two groups of MYB108A-suppressed grape calluses (suppressor 3 and suppressor 4) compared to the control (Fig. 7a); the melatonin-induced promotion of ACS1 expression and ethylene production was reduced in the suppressors (Fig. $7 \mathrm{~b}, \mathrm{c})$. Therefore, melatonin at least partially promoted ethylene production via MYB108A.

\section{MYB108A mediates melatonin-induced ethylene production and salt tolerance in 'Crimson seedless' calluses}

The grape calluses of suppressor 3 and suppressor 4, produced through transforming antisense cDNA fragments of the 3-UTR of MYB108A into grape calluses, were used to evaluate the role of $M Y B 108 A$ in melatonininduced salt tolerance. Under the control conditions, similar growth increments were found for WT and suppressor calluses. However, the growth of WT and suppressor calluses was completely inhibited by $100 \mathrm{mM}$ $\mathrm{NaCl}$ (Fig. 7d, e). In contrast, melatonin treatment alleviated the growth inhibition of WT and suppressor calluses caused by $\mathrm{NaCl}$ treatment. However, the suppressors still showed much smaller growth increments than the WT calluses under the treatment of $\mathrm{NaCl}$ plus melatonin (Fig. 7d, e). Additionally, the suppressors accumulated more MDA than the control under $\mathrm{NaCl}$ and $\mathrm{NaCl}$ plus melatonin (Fig. 7f), indicating that suppressors were more severely harmed by $\mathrm{NaCl}$ with or without melatonin. Taken together, melatonin-induced salt tolerance was negatively affected by the suppression of MYB108A. On the other hand, compared to the WT, suppressors possessed a reduced ACC content under all treatment conditions (Fig. $7 \mathrm{~g}$ ). Therefore, the suppression of $M Y B 108 A$ expression limited the effect of melatonin on ethylene production.

Taken together, melatonin alleviated salt injury via ethylene signaling, and this process was mediated at least partially by MYB108A. 

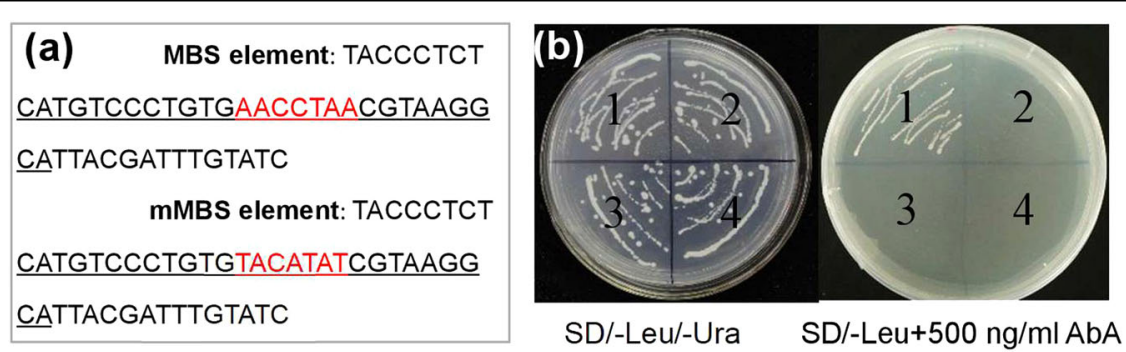

(c)

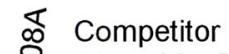
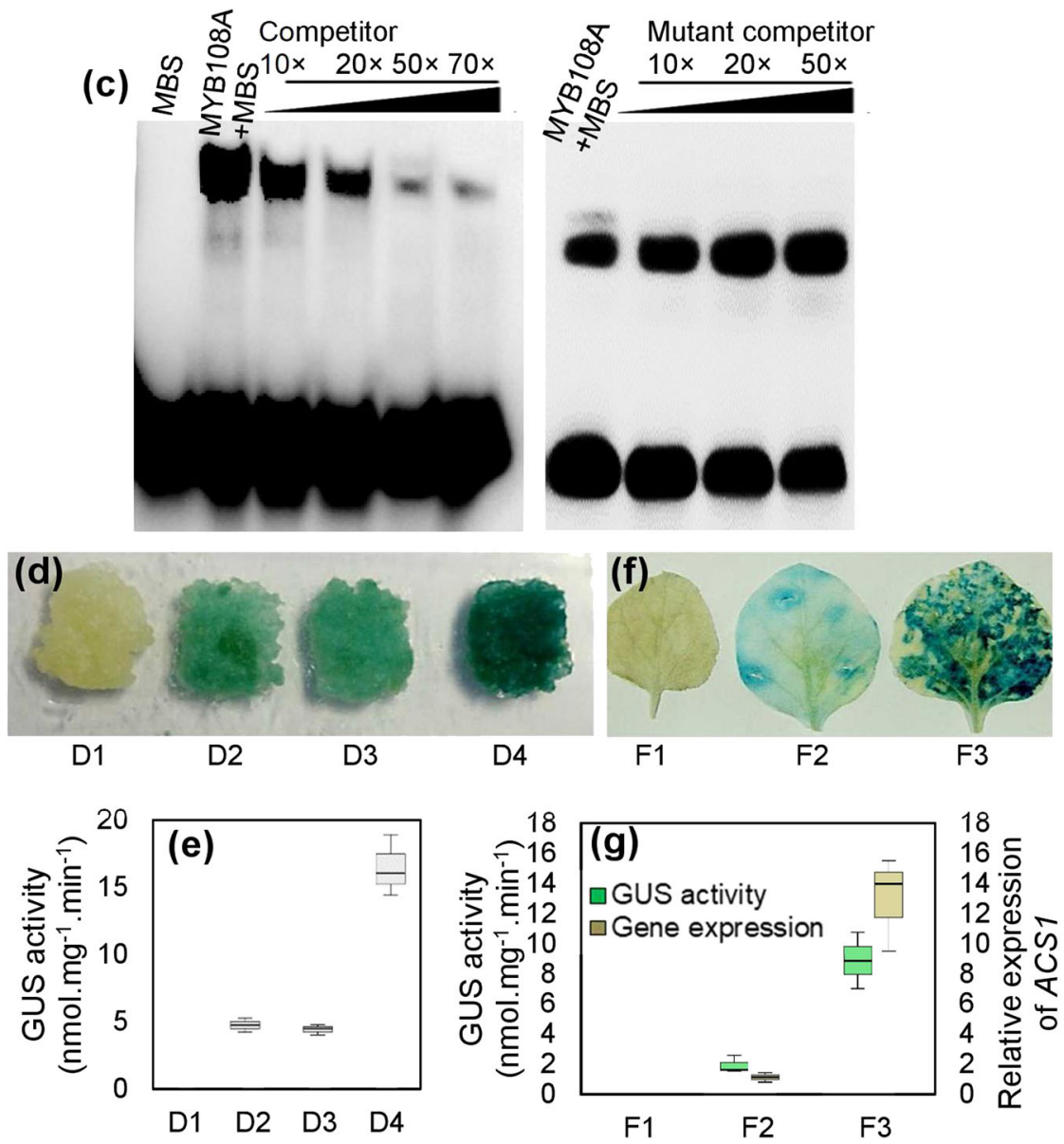

Fig. 5 Characterization of transcription activation of $A C S 1$ by MYB108A. a Sequences containing MBS or mMBS elements for yeast one-hybrid assay and underlined sequence for EMSA. b Yeast one-hybrid assay using MBS and mMBS as bait. (1) pAbAi-MBS/pGAD-MYB108A; (2) pAbAi-MBS/ pGAD7; (3) pAbAi-mMBS/pGAD-MYB108A; (4) pAbAi-mMBS/pGAD7. $\mathbf{c}$ Interaction of the MYB108A protein with labeled DNA probes for cis-elements of the ACS1 promoter in EMSA. $\mathbf{d}$, $\mathbf{f}$ Histochemical analysis of the transactivation activity of MYB108A via the binding MBS element. 'Crimson seedless' calluses $\mathbf{d}$ and tobacco leaves $\mathbf{f}$ were agro-infiltrated with different vector constructs: D1, WT calluses; D2, MBS-35S mini-GUS; D3, mMBS-35S miniGUS and 35S::MYB108A; D4, MBS-35S mini-GUS and 35S::MYB108A; F1, WT leaf; F2, PACS1::ACS1-GUS; F3, PACS1::ACS1-GUS and 35S::MYB108A. e GUS activities of grape calluses infiltrated by Agrobacterium containing D1-D4 constructs. $\mathbf{g}$ ACS1 expression and GUS activities of tobacco leaves infiltrated by Agrobacterium containing F1-F3 constructs. Each image is representative of three biological replicates in $\mathbf{b}, \mathbf{c}$, $\mathbf{d}$, and $\mathbf{f}$. In e and $\mathbf{g}$, values are presented as the means \pm SD of 3 replicates

\section{Discussion}

VviMYB108A participates in the melatonin-mediated signaling pathway in the salt stress response of 'Crimson seedless' grapevines

Many studies have demonstrated that melatonin plays a key role in abiotic stress tolerance in multiple species under exogenous application and endogenous induction $^{4,19}$. This study also indicated the role of melatonin in enhancing the salt tolerance of grapevines (Fig. 1). Additionally, rhizospheric treatment with melatonin increased melatonin levels not only in roots but also in leaves (Fig. 2a). Similarly, foliar or rhizospheric treatment with 

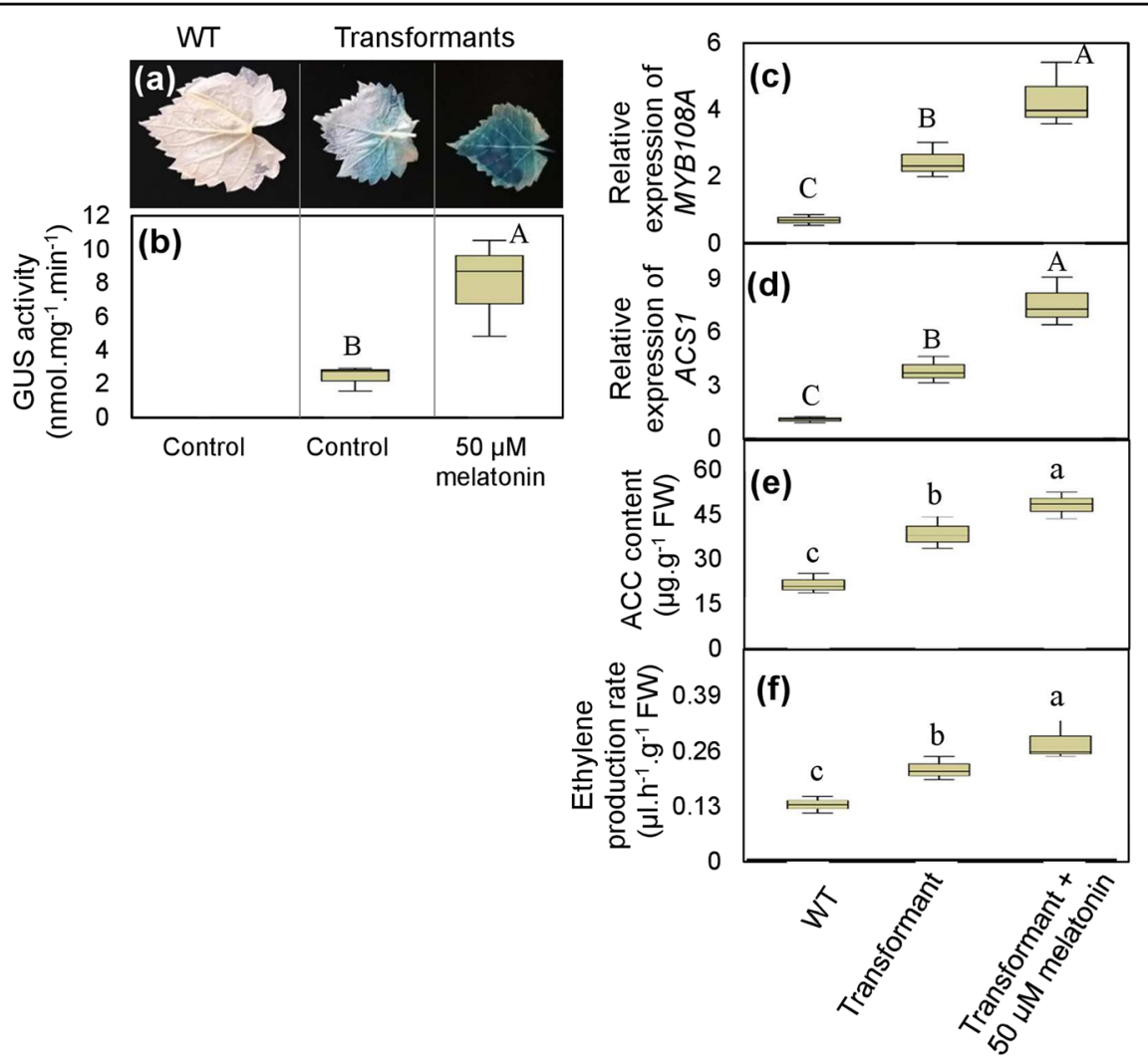

Fig. 6 Transient expression of MYB108A in response to melatonin and its effects on ACS1 expression, ACC and ethylene synthesis in in vitro grapevine leaves. Young 'Cabernet Sauvignon' vine leaves were infiltrated by Agrobacterium tumefaciens containing the construct $P_{\text {MYB108A: }}$ MYB108A-GUS. After 3 days of culture with or without $50 \mu \mathrm{M}$ melatonin, leaves were used for the determination of GUS staining a and activity $\mathbf{b}$, relative expression of MYB108A c and ACS1 d ACC content $\mathbf{e}$ and ethylene production rate $\mathbf{f}$. WT, wild type; transformant represents the grapevine

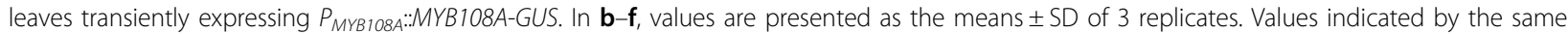
lowercase and capital letters are not significant at $P<0.05$ and $P<0.01$, respectively, on the basis of Duncan's multiple range test

melatonin increased the melatonin levels in untreated roots or leaves, respectively, under both normal and coldstress conditions ${ }^{29}$. Therefore, local melatonin application might regulate abiotic stress responses in untreated distant parts via long distance transport.

Under abiotic stresses, melatonin not only directly scavenges ROS as a powerful antioxidant but also acts as a signal molecule ${ }^{4}$. The mechanism underlying the melatonin-mediated signaling pathway in plant abiotic stress responses remains largely unknown, but melatonin confers plant abiotic stress tolerance by modulating relevant transcription factors ${ }^{19}$. For example, melatonin induces the transcription of $A t C B F s$ and confers enhanced resistance to abiotic stresses; additionally, the transcription factor AtZAT6-activated CBF pathway is essential for melatonin-mediated freezing stress resistance in Arabidopsis $^{30}$. Myb4 and AP37 are involved in melatoninmediated abiotic stresses ${ }^{31}$. Similarly, VviMYB108A mediated melatonin-induced salt tolerance (Fig. 7d-f).

In Arabidopsis, R2R3 MYBs are classified into 22 subgroups, and MYB108 and five other MYB proteins
(MYB2, MYB62, MYB78, MYB112 and MYB116) belong to subgroup $20^{32}$. Existing studies indicate that subgroup 20 is involved in abiotic stress responses. For example, AtMYB112 and AtMYB108 are significantly induced by salinity in Arabidopsis roots and are implicated in both biotic and abiotic stresses ${ }^{33,34}$. AtMYB2 mediates saltinduced $\mathrm{Ca}^{2+}$ signaling and results in salt tolerance in Arabidopsis plants ${ }^{35}$. In this study, VviMYB108A, as a member of subgroup 20, also conferred salt tolerance to grape calluses (Fig. S2; Fig. 7d-f). Similarly, the tomato MYB transcription factor SIAIM1, which is also clustered into subgroup 20, enhances resistance to abiotic stress by modulating responses to $\mathrm{ABA}^{36}$.

Taken together, VviMYB108A functions downstream of melatonin to enhance the salt tolerance of grapevines.

\section{VviMYB108A enhances salt tolerance of 'Crimson seedless' grapevines via ethylene}

Ethylene is well known as an important positive mediator in plant salt tolerance, such as grapevines (Fig. 1), maize $^{13}$ and tomato ${ }^{14}$. However, in other cases, ethylene 

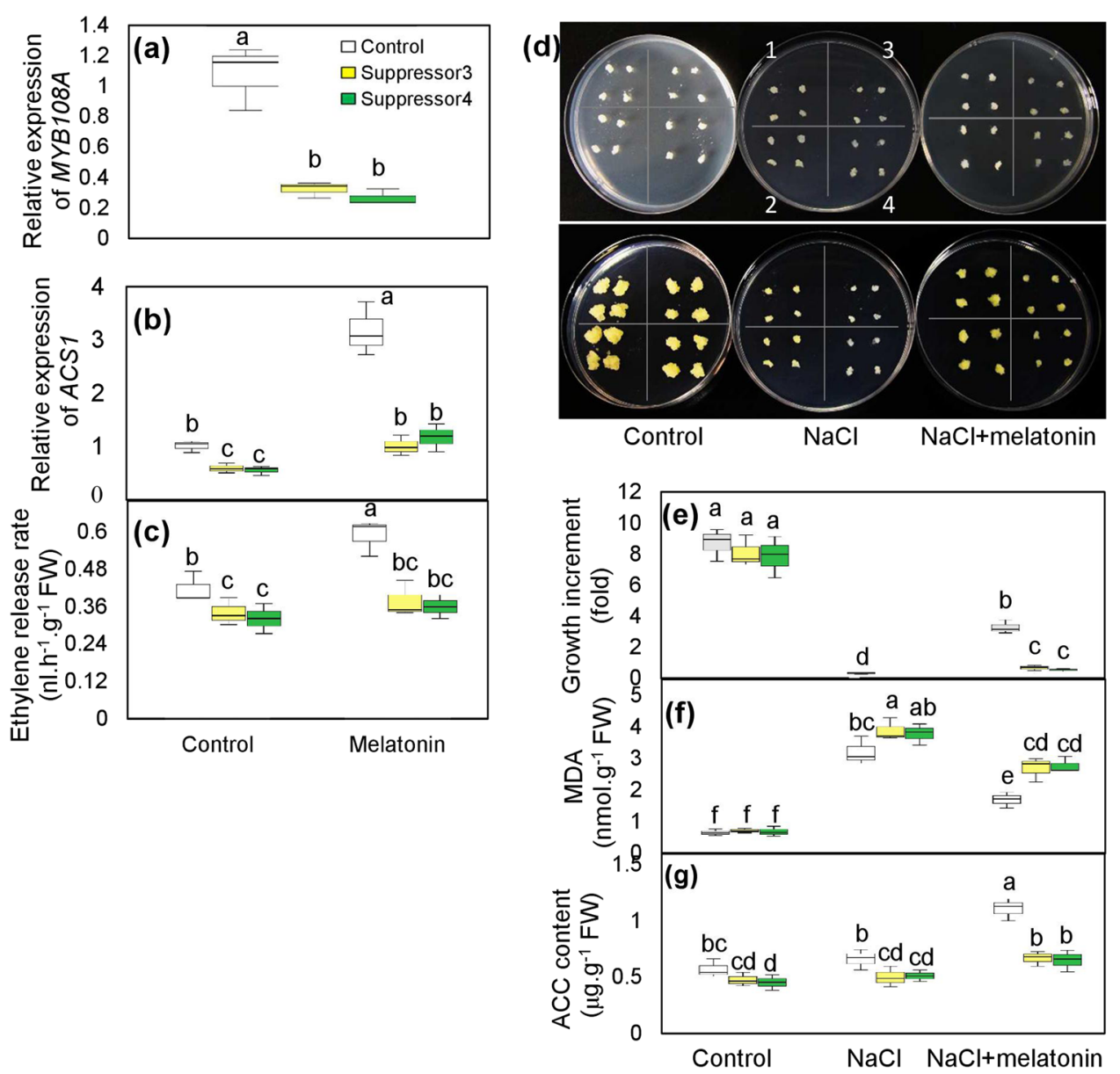

Fig. 7 Evaluation of salt tolerance of WT and MYB108A-suppressed 'Crimson seedless' calluses with or without melatonin. Expression of MYB108A a and ACS1 $\mathbf{b}$ and ethylene release rate $\mathbf{c}$ were determined using calluses at 21 days after subculture. $\mathbf{d}-\mathbf{g}$ Phenotype evaluation $\mathbf{d}$, growth increment e, MDA content $\mathbf{f}$ and ACC content $\mathbf{g}$ of WT and suppressor calluses treated with $100 \mathrm{mM} \mathrm{NaCl}$ with or without $50 \mu \mathrm{M}$ melatonin. In panel d, upper and lower photos were taken at 0 and 21 days after subculture, respectively; WT calluses were indicated by 1 and 2, and suppressor 3 and suppressor 4 were indicated by 3 and 4, respectively, for each culture dish; each image is representative of three biological replicates, and in other panels, different lowercase letters indicate significant difference on the basis of Duncan's multiple range test at $P<0.05$

levels can adversely affect salt tolerance. For instance, transgenic tobacco plants with poor ethylene biosynthesis exhibit elevated salt tolerance, and the treatment of rice plants with ethylene confers salt hypersensitivity ${ }^{15,37}$. Similarly, the role of ethylene in cold tolerance varies with plant species. For example, ethylene enhances cold tolerance in grapevine ${ }^{16}$; in contrast, ethylene negatively influences the cold tolerance of Medicago truncatula ${ }^{38}$. Therefore, the role of ethylene in abiotic stress tolerance is influenced by the plant species and even the developmental stage $^{39}$; additionally, fine-tuning ethylene biosynthesis may be essential to abiotic stress tolerance in plants ${ }^{7}$; moreover, homeostasis between ethylene and its receptors may affect the function of ethylene in salt stress ${ }^{40}$.

As a rate-limiting enzyme, ACS is the major target for the regulation of ethylene production under stress conditions $^{40}$. VviACS1 has been shown to respond to salt and to be responsible for ethylene biosynthesis (Fig. 1b; Fig. $4 \mathrm{f}-\mathrm{h}$ ). Similarly, $A C S 1 a$ and $A C S 1$ are the major genes responsive to salt stress in maize and tobacco, respectively $^{13,41}$. In addition to $A C S 1$, several other $A C S s$, such as $A C S 2$ and $A C S 7$, have also been reported to be induced by salt stress in Arabidopsis ${ }^{10}$. However, the Arabidopsis acs 7 mutant, which displays reduced ethylene production, exhibits increased salt tolerance at the seed germination stage $^{42}$, suggesting the complex regulation of ethylene synthesis catalyzed by ACSs in response to salt stress. In cotton, a series of ACSs are upregulated under both shortand long-term salinity conditions ${ }^{43}$. In this study, compared to the substantial decline in VvACS1 expression in the suppressors, a relatively small decrease in the ACC content and ethylene production rate was detected (Fig. $4 \mathrm{f}-\mathrm{h}$ ), suggesting the roles of other ACS isoforms in controlling ethylene synthesis in grapevines. Additionally, 
among the promoters of 21 ACS genes in soybean, all contain at least one MYB binding cis-acting element ${ }^{20}$, suggesting that their expression is regulated by MYBs. It was also verified that MYB1 overexpression increases ethylene production and upregulates the expression of ethylene synthetic genes, including ACS1, ACS3a, ACS4 and ACS6 in apple; in contrast, the results were obtained in the $M Y B 1$-suppressed tissues ${ }^{43}$, suggesting the transcriptional regulation of MYB1 on ACS expression. Additionally, VviMYB108A is coexpressed with VviERG1, VviERF113 and VviERF114, which are involved in ethylene signaling ${ }^{28}$. In this study, VviACS1 was identified to be directly transcriptionally activated by VviMYB108A (Fig. 5). Taken together, VviMYB108A promoted ethylene production by increasing VviACS1 expression and thereby enhanced the salt tolerance of grapevines.

\section{The regulatory effect of melatonin on ethylene synthesis may be related to complex hormone signal crosstalk}

In grapevines, melatonin enhanced the salt tolerance of vines and promoted berry ripening, which was related to ethylene production (Fig. 1) ${ }^{44}$. Additionally, VviACS1 was shown to be a target gene induced by melatonin (Fig. 5). In contrast, melatonin treatment promotes tomato postharvest ripening, and the ethylene production level correlates well with ACS4 expression ${ }^{45}$. However, melatonin treatment reduced ethylene production and resulted in delayed postharvest banana ripening and pear fruit senescence through the regulation of the expression of $A C O 1$ and $A C S 1^{46,47}$. In particular, melatonin treatment inhibits ethylene production in banana leaves, but combined treatments of melatonin and Fusarium wilt induce ethylene levels ${ }^{48}$. Therefore, it has been suggested that the function of melatonin in regulating ethylene biosynthesis may be indirect, and its positive or negative influence on ethylene production may be affected by other factors, possibly including additional signal molecules.

Recent studies have indicated that melatonin regulates sugar accumulation and metabolism in apple and tobacco plants ${ }^{49,50}$; changes in sugar status evoke the generation of sugar signals that are integrated with multiple hormone signaling pathways ${ }^{51}$; therefore, melatonin might act through interactions with sugar and hormone signaling pathways. Additionally, cumulative studies have shown that melatonin is an important modulator of gene expression related to plant hormones, such as IAA, ABA, gibberellins and ethylene ${ }^{24}$, and this modulation might play a key role in melatonin-mediated tolerance to abiotic stress. For example, melatonin promotes seed germination under high salt by regulating the $\mathrm{ABA}$ and GA4 interaction in cucumber ${ }^{52}$ and results in higher $A B A$ concentrations in drought-primed plants when exposed to cold stress ${ }^{53}$. However, ethylene biosynthesis is controlled via crosstalk with other hormones. For example, ethylene biosynthesis is tightly controlled by cytokinins ${ }^{54}$. In particular, our previous work demonstrated that melatonin treatment promoted ethylene production via $\mathrm{ABA}^{44}$. In this study, VviMYB108A promoted ethylene synthesis in response to melatonin (Fig. 6); additionally, AtMYB108 was also induced by ABA in Arabidopsis $\operatorname{roots}^{55}$. Therefore, it is suggested that melatonin possibly upregulates VviMYB108A through ABA.

Taken together, these results suggest that melatonin may regulate ethylene biosynthesis via complex crosstalk with other signal molecules and that the signaling molecules involved may at least partially determine the effect of melatonin on ethylene in a positive or negative manner. This hypothesis also explains why melatonin induced the expression of different $A C S$ genes in the above-mentioned species, and ACC and ethylene syntheses are not directly proportional to melatonin production (Fig. 2).

\section{Conclusions}

Melatonin and ethylene enhanced the salt tolerance of grapevines under the conditions applied in this study. Ethylene production was enhanced by melatonin, and ethylene participated in melatonin-induced salt tolerance. Further analysis revealed that MYB108A expression was strongly induced by melatonin, and MYB108A could directly bind to the promoter of $A C S 1$, activating its expression and promoting ethylene synthesis; MYB108A played a key role in the effect of melatonin on ethylene synthesis. Taken together, a pathway for melatonininduced salt tolerance was revealed, i.e., "melatoninMYB108A-ACS1-ethylene synthesis-salt tolerance".

\section{Materials and methods \\ Plant materials, growth conditions and experimental treatments}

'Crimson seedless' (Vitis vinifera) cuttings were used for salt tolerance assays. They were planted in $15-\mathrm{cm}-$ diameter plastic pots filled with a 2:1 (v/v) mixture of soil:vermiculite in a greenhouse under normal conditions. For the salt assay, vine cuttings were watered every 3 days with $100 \mathrm{mM} \mathrm{NaCl}$ in the presence or absence of $50 \mu \mathrm{M}$ melatonin, $50 \mu \mathrm{M}$ ACC and $2 \mu \mathrm{M}$ AVG.

'Crimson seedless' in vitro shoot cultures were used for the determination of melatonin, ACC, ethylene production rate and gene expression. Five-week-old uniform vines were treated with Hoagland's nutrient solution (control) and $50 \mu \mathrm{M}$ melatonin in glass bottles with a $10-\mathrm{cm}$ height and 6-cm diameter. Each glass bottle was provided with sufficient oxygen with an oxygen machine (SenSen Group, China). The vines were grown in a controlled-environment growth cabinet with a temperature of $25^{\circ} \mathrm{C}$, a 14 -h photoperiod and a light intensity of $600 \mu \mathrm{mol} / \mathrm{m}^{2} / \mathrm{s}$. 
'Crimson seedless' grape calluses were used for gene transformation and the salt tolerance assay. The callus was subcultured on MS medium supplemented with $0.59 \mathrm{~g} / \mathrm{L}$ 2-(N-Morpholino) ethanesulfonic acid, $10 \mathrm{mg} / \mathrm{L}$ picloram, and $2.2 \mathrm{mg} / \mathrm{L}$ thidiazuron at $25^{\circ} \mathrm{C}$ under dark conditions. For the salt assay, MS medium was supplemented with $100 \mathrm{mM} \mathrm{NaCl}$ alone or combined with $50 \mu \mathrm{M}$ melatonin.

The young leaves of 'Cabernet Sauvignon' grapevines cultivated in the field were used for transient transformation. The leaves of Nicotiana benthamiana seedlings were used for the transformation of $A M S T$. They were planted in 10-cm-dimeter plastic pots with culture stroma and grown under a 16 -h light/8-h dark photoperiod at approximately $600 \mu \mathrm{mol} / \mathrm{m}^{2} / \mathrm{s}$ and $28^{\circ} \mathrm{C}$.

\section{Determination of the root activity, relative electrical conductivity and malondialdehyde (MDA) content}

Root activity was measured using the triphenyl tetrazolium chloride (TTC) method ${ }^{56}$. The relative electric conductivity was measured and calculated as described by Zhou and Leul ${ }^{57}$. MDA was determined using the thiobarbituric acid reactive substances assay, as previously reported $^{58}$.

\section{Determinations of melatonin, ACC and ethylene production rate}

Melatonin was extracted according to our previous study $^{18}$. The samples were separated on a Waters Acquity UHPLC system (Milford, MA, USA) equipped with a BEH $\mathrm{C}_{18}$ column (Waters, $2.1 \mathrm{~mm}$ internal diameter $\times 50 \mathrm{~mm}$ length, and $1.7 \mu \mathrm{m}$ particle size). Mass spectrometry (MS) analyses were performed using a QTof-Micro mass spectrometer. The detection parameters and conditions of the ultra-high-performance liquid chromatography (UHPLC)MS analysis were set according to our previous study ${ }^{26}$.

The ethylene production rate was measured as described in a previous study ${ }^{18}$. ACC extraction and determination were performed according to the method described by Tucker et al. ${ }^{59}$.

\section{RNA extraction and quantitative RT-PCR}

Total RNA was isolated using RNA plant Plus Reagent (Tiangen, Beijing, China). qRT-PCR was performed using the Ultra SYBR Mixture (SYBR Green I) (CWBIO, Beijing, China) in an ABI7500 qRT-PCR instrument (ABI, MA, USA) according to the manufacturer's instructions. $V v U B I$ was used as the internal reference. The specific primers of the amplified genes are listed in Supplementary Table S1.

\section{Subcellular localization of the MYB108A protein}

The MYB108A open reading frame was isolated and cloned into the binary vector pROKII-GFP downstream of the $35 S$ promoter. The resultant construct 35S::MYB108A-
GFP was introduced into Agrobacterium tumefaciens GV3101 and transformed into onion epidermal cells ${ }^{60}$ and leaves from 5 -week-old $N$. benthamiana seedlings ${ }^{61}$. After 2-3 days of incubation, the subcellular localization of GFP was monitored with an epifluorescence microscope (Olympus BX53F, Tokyo, Japan).

\section{Transformation of MYB108A, ACS1 and ASMT into grape calluses and/or tobacco plants}

The complete coding regions of MYB108A and ASMT, used for sense overexpression, were isolated from 'Crimson seedless' roots and cloned into the pRI101-AN (Takara, Dalian, China) vector downstream of the $35 \mathrm{~S}$ promoter. The 3-UTR sequences of MYB108A and ACS1 were cloned into the same vector for antisense suppression. The resultant constructs were introduced into Agrobacterium strain LBA4404 and transformed into grape callus, as described by Li et al..$^{62}$ with some modifications, and into tobacco leaves, as reported by Wang et al. ${ }^{63}$. For grape callus transformation, grape calluses were immersed in an Agrobacterium suspension for $20 \mathrm{~min}$, blotted dry on sterile filter paper and transferred to solid MS medium with $100 \mu \mathrm{M}$ acetosyringone. After two days of coculture in darkness at $25^{\circ} \mathrm{C}$, the calluses were transferred to the screening medium supplemented with $100 \mathrm{mg} / \mathrm{L}$ kanamycin and $300 \mathrm{mg} / \mathrm{L}$ cefalexin. Five weeks later, most of the calluses had died, and the obtained calluses were subcultured on screening medium at 4-week intervals. The ACS1- and MYB108A-suppressed grape calluses from two independent Agrobacteriummediated transformations were obtained and designated as suppressors 1 and 2 and suppressors 3 and 4, respectively.

\section{Binding assays of MYB108A to the MBS element using a yeast one-hybrid system and EMSA}

For the yeast one-hybrid assay, the MBS element was synthesized and inserted into the pAbAi vector. The ORF of $M Y B 108 A$ was amplified and fused in-frame with the GAL4 activation domain of the pGADT7 vector. The mutant MBS (mMBS) was used as a negative control. The resultant plasmid was introduced into the yeast strain Y1HGold. The detailed procedure was performed according to the user manual for the Matchmaker Gold Yeast One-Hybrid Library Screening System (Clontech, Mountain View, CA, USA).

For EMSA, the MYB108A ORF was cloned into the expression vector pEASY-E1 (TransGenBiotech, Beijing, China). The MYB108A-His recombinant protein was expressed in Escherichia coli strain BL21 and purified using HIS-tag BeaverBeads ${ }^{\text {tix }}$ Nickel (Beaver, BioBAY, China). The ACS1 promoter probe containing an MBS element was synthesized and labeled with biotin (Sangon, Shanghai, China). Unlabeled competitor probes were generated from the dimerized oligos of the ACS1 promoter regions 
containing the MBS element. EMSA was performed as described in the instruction manual included with the EMSA Kit (Thermo Fisher Scientific, MA, USA).

\section{Assay of the MYB108A promoter activity}

The 1872-bp promoter of MYB108A, used for expression response, was isolated and fused to pRI101-GUS via replacing its $35 S$ promoter $\left(P_{M Y B 108 A}:: G U S\right)$. The resultant construct was introduced into Agrobacterium strain LBA4404 and transformed into grape callus, as described above. GUS histochemical staining was performed according to the methods of Jefferson et al. ${ }^{64}$. The GUS activity was calculated as nmol of 4-methylumbelliferone (4-MU) per mg protein per minute.

Transient transformation of $M Y B 108 A$ into different tissues

The complete coding region of $M Y B 108 A$ was inserted upstream of GUS in the construct $P_{M Y B 108 A}:: G U S$, and the resultant construct was designated $P_{M Y B 108 A}:$ : MYB108A-GUS. The constructed plasmids were introduced into Agrobacterium strain GV3101. The Agrobacterium-mediated transient transformation of 'Cabernet Sauvignon' leaves was performed, as previously described ${ }^{65}$.

Transient cotransformation was used to determine whether MYB108A could bind to the MBS element and activate the expression of downstream genes. The synthesized sequences containing MBS and mMBS were fused upstream of the $35 \mathrm{~S}$ minimal promoter of pRI101GUS (Takara, Dalian, China) to generate the MBS and mMBS mini-GUS plasmids. The promoter sequence of $A C S 1,1500$ bp upstream of ATG, was used to replace the $35 \mathrm{~S}$ promoter of pRI101-GUS, and the ACS1 ORF was inserted upstream of GUS, generating the $P_{A C S 1}: A C S 1$ GUS plasmid. The above two plasmids and the plasmid of $35 S:: M Y B 108 A$, constructed above for the sense overexpression of $M Y B 108 A$, were introduced into Agrobacterium strain GV3101. The Agrobacteriummediated transient transformation of tobacco leaves was performed according to Yang et al. ${ }^{66}$. The Agrobacterium-mediated transient transformation of grape calluses was performed according to the same procedure of infiltration and coculture in genetic transformation of calluses mentioned above.

\section{Transactivation property assay of MYB108A}

The yeast two-hybrid system was employed to determine the transcription activation property of MYB108A as a transcription factor. The complete $M Y B 108 A$-coding sequence, $\triangle M Y B 108 A /-51 a a$ or $\triangle M Y B 108 A /-102 a a$ was isolated and cloned into pGBKT7 to generate an in-frame fusion with the GAL4 DNA-binding domain. The plasmid pGBKT7-MYB108A, pGBKT7- $\triangle$ MYB108A/-51aa or pGBKT7- $\triangle$ MYB108A/-102aa was transformed into yeast. SD media supplemented with -Trp/-His/-Ade and 5-bromo-4-chloro-3-indolyl-d-galactopyranoside acid ( $\mathrm{x}$ $\alpha$-gal) was used to test for possible transcription activation.

\section{Statistical Analysis}

All statistical analyses were performed by SPSS (V19.0) software. A one-way analysis of variance (ANOVA) followed by Duncan's multiple range test and/or nonparametric Kruskal-Wallis test were employed, and standard deviation (SD) was calculated from three replicates.

\section{Acknowledgements}

This study was supported by the National Natural Science Foundation of China (31872068), the Natural Science Foundation of Shandong Province (ZR2018MC021), the Funds of Shandong "Double Tops" Program (SYL2017YSTD10) and China's Agricultural Research System (CARS-29).

\section{Author contributions}

Yu-Xin Yao and Li-Li Xu conceived and designed the experiments. Li-Li Xu Guang-Qing Xiang, Qing-Hua Sun, Yong Ni and Shi-Wei Gao carried out the experiments. Zhong-Xin Jin performed the data analysis. Yu-Xin Yao wrote the paper.

\section{Data availability}

Data supporting the results can be found in this paper.

Conflict of interest

The authors declare that they have no conflict of interest.

Supplementary Information accompanies this paper at (https://doi.org/ 10.1038/s41438-019-0197-4).

Received: 18 February 2019 Revised: 3 July 2019 Accepted: 14 August 2019 Published online: 08 October 2019

\section{References}

1. Munns, R. \& Tester, M. Mechanisms of salinity tolerance. Annu. Rev. Plant Biol. 59, 651-681 (2008)

2. Walker, R. R., Blackmore, D. H. \& Clingeleffer, R. L. Rootstock effects of salt tolerance of irrigated field-grown grapevines (Vitis vinifera L. CV. Sultana) I. Yield and vigour inter-relationships. Aust. J. Grapevine Wine Res. 8, 3-14 (2002).

3. Ryu, H. \& Cho, Y. G. Plant hormones in salt stress tolerance. J. Plant Biol. 58, 147-155 (2015)

4. Arnao, M. B. \& Hernandez, R. J. Functions of melatonin in plants: a review. J. Pineal Res. 59, 133-150 (2015).

5. Nguyen, D. et al. How plants handle multiple stresses: hormonal interactions underlying responses to abiotic stress and insect herbivory. Plant Mol. Biol. 91, 727-740 (2016).

6. Nemhauser, J. L., Hong, F. \& Chory, J. Different plant hormones regulate similar processes through largely nonoverlapping transcriptional responses. Cell $\mathbf{1 2 6}$ 467-475 (2006).

7. Zhang, M., Smith, J. A. C., Harberd, N. P. \& Jiang, C. The regulatory roles of ethylene and reactive oxygen species (ROS) in plant salt stress responses. Plant Mol. Biol. 91, 651-659 (2016).

8. Pierik, R., Tholen, D., Poorter, H., Visser, E. J. W. \& Voesenek, L. A. C. J. The Janus face of ethylene: growth inhibition and stimulation. Trends Plant Sci. 11, 176-183 (2006).

9. Argueso, C. T., Hansen, M. \& Kieber, J. J. Regulation of ethylene biosynthesis. J. Plant Growth Regul. 26, 92-105 (2007).

10. Achard, P. et al. Integration of plant responses to environmentally activated phytohormonal signals. Science 311, 91-94 (2006). 
11. Dong, $\mathrm{H}$. et al. Loss of ACS7 confers abiotic stress tolerance by modulating ABA sensitivity and accumulation in Arabidopsis. J. Exp. Bot. 62, 4875-4887 (2011).

12. Yang, L., Zu, Y. G. \& Tang, Z. H. Ethylene improves Arabidopsis salt tolerance mainly via retaining $\mathrm{K}^{+}$in shoots and roots rather than decreasing tissue $\mathrm{Na}^{+}$ content. Environ. Exp. Bot. 86, 60-69 (2013).

13. Freitas, V. S. et al. Ethylene triggers salt tolerance in maize genotypes by modulating polyamine catabolism enzymes associated with $\mathrm{H} 2 \mathrm{O} 2$ production. Environ. Exp. Bot. 145, 75-86 (2017).

14. Gharbi, E. et al. Inhibition of ethylene synthesis reduces salt-tolerance in tomato wild relative species Solanum chilense. Plant Physiol. 210, 24-37 (2017)

15. Müller, M. \& Munnébosch, S. Ethylene response factors. A key regulatory hub in hormone and stress signaling. Plant Physiol. 169, 32-41 (2015).

16. Sun, $X$. et al. Ethylene positively regulates cold tolerance in grapevine by modulating the expression of ETHYLENE RESPONSE FACTOR 057. Sci. Res. 6 , 24066-24079 (2016).

17. Arraes, F. B. et al. Implications of ethylene biosynthesis and signaling in soybean drought stress tolerance. BMC Plant Biol. 15, 213-232 (2015).

18. $\mathrm{Xu}$, L. et al. Melatonin enhances phenolics accumulation partially via ethylene signaling and resulted in high antioxidant capacity in grape berries. Front Plant Sci. 8, 1426-1437 (2017).

19. $Y u, Y$. et al. The role of phyto-melatonin and related metabolites in response to stress. Molecules 23, 1887-1901 (2018).

20. Zuo, B. et al. Overexpression of MzASMT improves melatonin production and enhances drought tolerance in transgenic Arabidopsis thaliana plants. J. Pineal Res. 57, 408-417 (2015).

21. Li, C. et al. The mitigation effects of exogenous melatonin on salinity-induced stress in Malus hupehensis. J. Pineal Res. 53, 298-306 (2012).

22. Arnao, M. B. \& Hernández-Ruiz, J. Melatonin and its relationship to plant hormones. Ann. Bot. 121, 195-207 (2017).

23. Li, X. et al. Melatonin enhances cold tolerance in drought-primed wild-type and abscisic acid-deficient mutant barley. J. Pineal Res. 61, 328-339 (2016).

24. Li, C. et al. Melatonin mediates the regulation of ABA metabolism, free-radical scavenging, and stomatal behaviour in two Malus species under drought stress. J. Exp. Bot. 66, 669-680 (2015).

25. Lee, H. Y. \& Back, K. Mitogen-activated protein kinase pathways are required for melatonin-mediated defense responses in plants. J. Pineal Res. 60, 327-335 (2016).

26. Zhao, G. et al. Nitric oxide is required for melatonin-enhanced tolerance against salinity stress in rapeseed (Brassica napus L.) Seedlings. Int. J. Mol. Sci. 19, 1912-1931 (2018).

27. Kang, K. et al. Molecular cloning of a plant $\mathrm{N}$-acetylserotonin methyltransferase and its expression characteristics in rice. J. Pineal Res. 50, 304-309 (2011).

28. Wong, D. C. J. et al. A systems-oriented analysis of the grapevine R2R3-MYB transcription factor family uncovers new insights into the regulation of stilbene accumulation. DNA Res. 23, 451-466 (2016).

29. Li, H. et al. Local melatonin application induces cold tolerance in distant organs of Citrullus lanatus L. via long distance transport. Sci. Res. 7, 40858-40872 (2017)

30. Shi, H. \& Chan, Z. The cysteine2/histidine2-type transcription factor ZINC FINGER OF ARABIDOPSIS THALIANA 6-activated C-REPEAT-BINDING FACTOR pathway is essential for melatonin-mediated freezing stress resistance in Arabidopsis. J. Pineal Res. 57, 185-191 (2014).

31. Lee, H. Y. \& Back, K. 2-hydroxymelatonin promotes the resistance of rice plant to multiple simultaneous abiotic stresses (combined cold and drought). J. Pineal Res. 61, 303-316 (2016).

32. Lotkowska, M. E. Functional analysis of MYB112 transcription factor in the model plant Arabidopsis thaliana. Text.thesis.doctoral. https://publishup.unipotsdam.de/frontdoor/index/index/docld/6995 (2014).

33. Singh, K., Singla-Pareek, S. L. \& Pareek, A. Dissecting out the crosstalk between salinity and hormones in roots of Arabidopsis. OMICS 15, 913-924 (2011).

34. Mokhtari, F., Rafiei, F., Shabani, L. \& Shiran, B. Differential expression pattern of transcription factors across annual Medicago genotypes in response to salinity stress. Biol. Plantarum 61, 227-234 (2017).

35. Yoo, J. H. et al. Direct interaction of a divergent CaM isoform and the transcription factor, MYB2, enhances salt tolerance in Arabidopsis. J. Biol. Chem. 280, 3697-3706 (2005)

36. AbuQamar, S., Luo, H., Laluk, K., Mickelbart, M. V. \& Mengiste, T. Crosstalk between biotic and abiotic stress responses in tomato is mediated by the AIM1 transcription factor. Plant J. 58, 347-360 (2009).
37. Yang, $C$. et al. MAOHUZI6/ETHYLENE INSENSITIVE3-LIKE1 and ETHYLENE INSENSITIVE3-LIKE2 regulate ethylene response of roots and coleoptiles and negatively affect salt tolerance in rice. Plant Physiol. 169, 148-165 (2015).

38. Zhang, Z. \& Huang, R. Enhanced tolerance to freezing in tobacco and tomato overexpressing transcription factor TERF2/LeERF2 is modulated by ethylene biosynthesis. Plant Mol. Biol. 73, 241-249 (2010).

39. Kazan, K. Diverse roles of jasmonates and ethylene in abiotic stress tolerance. Trends Plant Sci. 20, 219-229 (2015).

40. Tao, J. J. et al. The role of ethylene in plants under salinity stress. Front Plant Sci. 6, 1059-1070 (2015).

41. Cao, W. H. et al. Expression of tobacco ethylene receptor NTHK1 alters plant responses to salt stress. Plant Cell Environ. 29, 1210-1219 (2006).

42. Dong, $\mathrm{H}$. et al. Loss of ACS7 confers abiotic stress tolerance by modulating ABA sensitivity and accumulation in. Arabidopsis. J. Exp. Bot. 62, 4875-4887 (2011).

43. An, J. P. et al. EIN3-LIKE1, MYB1, and ETHYLENE RESPONSE FACTOR3 act in a regulatory loop that synergistically modulates ethylene biosynthesis and anthocyanin accumulation. Plant Physiol. https://doi.org/10.1104/pp.18.00068 (2018).

44. Xu, L., Yue, Q., Xiang, G., Bian, F. \& Yao, Y. Melatonin promotes ripening of grape berry via increasing the levels of $A B A, \mathrm{H}_{2} \mathrm{O}_{2}$, and particularly ethylene. Horti. Res. 5, 41-51 (2018).

45. Sun, Q. Q. et al. Melatonin promotes ripening and improves quality of tomato fruit during postharvest life. J. Exp. Bot. 66, 657-668 (2015).

46. Hu, W. et al. Natural variation in banana varieties highlights the role of melatonin in postharvest ripening and quality. J. Agr. Food Chem. 65, 9987-9994 (2017).

47. Zhai, R. et al. Melatonin limited ethylene production, softening and reduced physiology disorder in pear (Pyrus communis L.) fruit during senescence. Postharvest Biol. Tec. 139, 38-46 (2018).

48. Wei, Y. X. et al. Identification, transcriptional and functional analysis of heatshock protein 90 s in banana (Musa acuminata L.) highlight their novel role in melatonin-mediated plant response to Fusarium wilt. J. Pineal Res. 62, 1-12 (2017).

49. Kobylińska, A., Borek, S. \& Posmyk, M. M. Melatonin redirects carbohydrates metabolism during sugar starvation in plant cells. J. Pineal Res. 64, e12466 (2018).

50. Yang, J. J. et al. Melatonin-mediated sugar accumulation and growth inhibition in apple plants involves down-regulation of fructokinase 2 expression and activity. Front. Plant Sci. 10, 150 (2019).

51. Ljung, K., Nemhauser, J. L. \& Perata, P. New mechanistic links between sugar and hormone signalling networks. Curr. Opin. Plant Biol. 25, 130-137 (2015).

52. Zhang, H. J. et al. Melatonin promotes seed germination under high salinity by regulating antioxidant systems, $A B A$ and $G A(4)$ interaction in cucumber (Cucumis sativus L.). J. Pineal Res. 57, 269-279 (2014).

53. Li, C. et al. Exogenous melatonin improved potassium content in Malus under different stress conditions. J. Pineal Res. 61, 218-229 (2016).

54. Zdarska, M. et al. Illuminating light, cytokinin, and ethylene signalling crosstalk in plant development. J. Exp. Bot. 66, 4913-4931 (2015).

55. Fujita, Y., Fujita, M., Shinozaki, K. \& Yamaguchi-Shinozaki, K. ABA-mediated transcriptional regulation in response to osmotic stress in plants. J. Plant Res. 124, 509-525 (2011).

56. Zhang, X., Huang, G., Bian, X. \& Zhao, Q. Effects of root interaction and nitrogen fertilization on the chlorophyll content, root activity, photosynthetic characteristics of intercropped soybean and microbial quantity in the rhizosphere. Plant Soil Environ. 59, 80-88 (2013).

57. Zhou, W. J. \& Leul, M. Uniconazole-induced alleviation of freezing injury in relation to changes in hormonal balance, enzyme activities and lipid peroxidation in winter rape. Plant Growth Regul. 26, 41-47 (1998).

58. Hodges, D. M., DeLong, J. M., Forney, C. F. \& Prange, R. K. Improving the thiobarbituric acid-reactive-substances assay for estimating lipid peroxidation in plant tissues containing anthocyanin and other interfering compounds. Planta 207, 604-611 (1999).

59. Tucker, M. L., Xue, P. \& Yang, R. 1-Aminocyclopropane-1-carboxylic acid (ACC) concentration and ACC synthase expression in soybean roots, root tips, and soybean cyst nematode (Heterodera glycines)-infected roots. J. Exp. Bot. 61, 463-472 (2010).

60. Ben-Romdhane, W. et al. Overexpression of AlTMP2 gene from the halophyte grass Aeluropus littoralis in transgenic tobacco enhances tolerance to different abiotic stresses by improving membrane stability and deregulating some stress-related genes. Protoplasma 255, 1161-1177 (2018). 
61. Nobumitsu, S., Eita, T. \& Hiroshi, N. Altered Subcellular localization of a tobacco membrane raft-associated remorin protein by tobamovirus infection and transient expression of viral replication and movement proteins. Front. Plant Sci. 9, 619-634 (2018).

62. Li, D. D., Shi, W. \& Deng, X. X. Agrobacterium-mediated transformation of embryogenic calluses of Ponkan mandarin and the regeneration of plants containing the chimeric ribonuclease gene. Plant Cell Rep. 21, 153-156 (2002).

63. Wang, F., Wang, C., Yan, Y., Jia, H. \& Guo, X. Overexpression of cotton GhMPK11 decreases disease resistance through the gibberellin signaling pathway in transgenic Nicotiana benthamiana. Front Plant Sci. 7, 689-704 (2016).
64. Jefferson, R. A., Kavanagh, T. A. \& Bevan, M. W. GUS fusions, beta-glucuronidase as a sensitive and versatile gene fusion marker in higher plants. EMBO J. $\mathbf{6}$ 3901-3907 (1987).

65. Lizamore, D. \& Winefield, C. The addition of an organosilicone surfactant to Agrobacterium suspensions enables efficient transient transformation of in vitro grapevine leaf tissue at ambient pressure. Plant Cell Tiss. Org. 120 607-615 (2015).

66. Yang, Y., Li, R. \& Qi, M. In vivo analysis of plant promoters and transcription factors by agroinfiltration of tobacco leaves. Plant J. 22, 543-551 (2000). 